\title{
A Case of IgG4-related Mesenteritis and Ankylosing Spondylitis
}

\author{
Ozlem Pehlivan ${ }^{1}$, Sibel Ocak Serin ${ }^{2}$ and Ezgi Ersoy Yesil ${ }^{3}$ \\ ${ }^{1}$ Department of Rheumatology, University of Health Sciences, Umraniye Training and Research Hospital, Istanbul, Turkey \\ ${ }^{2}$ Department of Internal Medicine, University of Health Sciences, Umraniye Training and Research Hospital, Istanbul, Turkey \\ ${ }^{3}$ Department of Nephrology, University of Health Sciences, Umraniye Training and Research Hospital, Istanbul, Turkey
}

\begin{abstract}
Immunoglobulin G4-related disease (IgG4-RD) is a systemic fibroinflammatory disease of unknown etiology and pathogenesis, which can affect all organs. The most commonly involved organs are the pancreas, hepatobiliary system, salivary glands, orbits and lymph nodes. Rarely, the thyroid, pituitary, aorta, lung and kidney may also be involved; mesenteric involvement is rare. The association of IgG4-RD with some rheumatological diseases is observed; while there are data in the literature about rheumatoid arthritis and Sjögren syndrome, but association with ankylosing spondylitis is rare. Anti-tumor necrosis factor alpha (anti-TNF $\alpha$ ) treatment has been tried in refractory IgG4-RD cases; but this case is of great interest in terms of progressive presentation under treatment with etanercept, an anti-TNF $\alpha$ agent, which is also used for the treatment. But, IgG4-related mesenteritis with ankylosing spondylitis receiving anti-TNF $\alpha$ therapy, which has also been tried in the treatment of IgG4 disease, has not been reported in the literature.
\end{abstract}

Key Words: IgG4-related disease, Mesenteritis, Ankylosing spondylitis.

How to cite this article: Pehlivan O, Serin SO, Yesil EE. A Case of IgG4-related Mesenteritis and Ankylosing Spondylitis. J Coll Physicians Surg Pak 2022; 32(01):111-113.

\section{INTRODUCTION}

Immunoglobulin G4-related disease (IgG4-RD) is a chronic inflammatory syndrome with an imprecisely elucidated pathogenesis. It is characterised by the presence of tumoral mass lesions, storiform fibrosis, obliterative phlebitis, and IgG4 producing plasma cells in the affected organs. ${ }^{1}$ The most commonly involved organs are the pancreas, hepatobiliary system, salivary glands, orbits and lymph nodes. Mesenteric involvement is rare. ${ }^{2}$

There are data in the literature on association of this syndrome with rheumatoid arthritis (RA) and Sjögren syndrome (SS), but association with ankylosing spondylitis (AS) is rare. ${ }^{1,2}$ We, herein, present a patient receiving anti-TNF $\alpha$ for AS, who progressed under treatment and was diagnosed with IgG4-related sclerosing mesenteritis.

Correspondence to: Dr. Sibel Ocak Serin, Department of Internal Medicine, University of Health Sciences, Umraniye Training and Research Hospital, Istanbul, Turkey

E-mail: rdsibelocak@gmail.com

Received: December 04, 2020; Revised: April 26, 2021;

Accepted: June 01, 2021

DOI: https://doi.org/10.29271/jcpsp.2022.01.111

\section{CASE REPORT}

A 38-year male was diagnosed with HLA B27-positive AS, according to the Modified New York criteria in 2015; and anti-T$N F-\alpha$ agent, etanercept, was started three years earliar, as there was no response to sulfasalazine $2 \times 2$ and non-steroidal antiinflammatory therapy. Grade I hydronephrosis was observed in the right kidney during $15^{\text {th }}$ month of etanercept treatment on ultrasonography obtained due to swelling in both testicles and abdominal pain. Abdominal computed tomography (CT) revealed a mass of $4.5 \mathrm{~cm}$ in diameter, with hyperdense soft tissue density, in the medial aspect of the ascending colon (Figure 1). The patient's ESR and CRP levels were $85 \mathrm{~mm} / 1^{\text {st }} \mathrm{h}$ and $6.7 \mathrm{mg} / \mathrm{dl}$, respectively. Anti-TNF $\alpha$ treatment was discontinued and the patient was operated with a preliminary diagnosis of colon malignancy. Intraoperatively, a hard, fixed mass of $4 \times 5 \mathrm{~cm}$ was observed to extend behind the cecum to the mesentery. Gonadal structures entered the mass, with which the ureter was closely related. The mass involved the ileocolic mesentery in fullthickness and extended to the inferior vena cava. Complete resection of the mass along with right hemicolectomy was performed. Pathological examination revealed lymphoid follicles, increased plasma cells, obliterative phlebitis and multinuclear giant cells, accompanied by pronounced fibrosis in the mesenteric fattissue. CD20, CD3, and Ki-67 were positive in scattered cells on immunohistochemistry. IgG4 positive cells were on average 70/high power field (HPF). The IgG4 (+)/lgG (+) cell ratio was $64 \%$, and vascular structures were CD34 positive on immunostaining. 


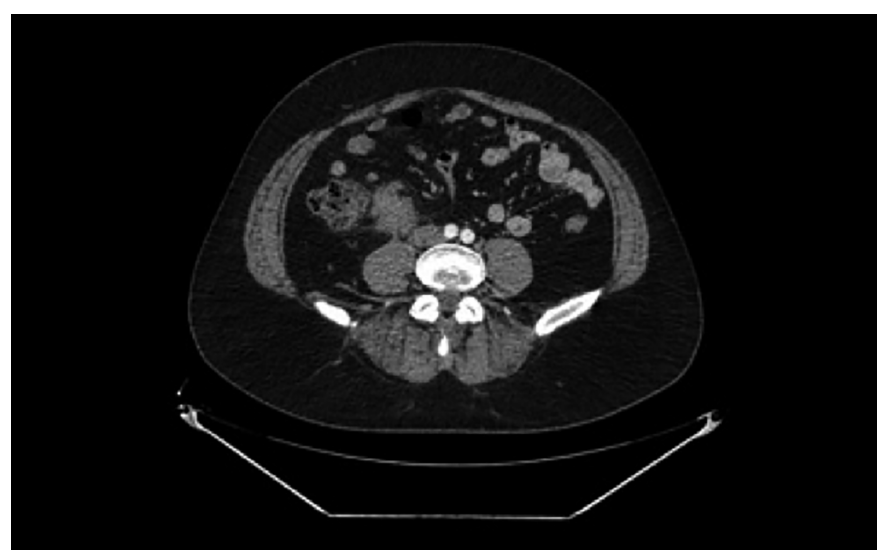

Figure 1: Abdominal computed tomography (CT) image after intravenous contrast reveals an irregular-shaped mass lesion (arrow), occupying the ileocecal mesentery with minimum contrast enhancement. The lesion envelops the bowel wall with narrowing of the intestinal cavity.

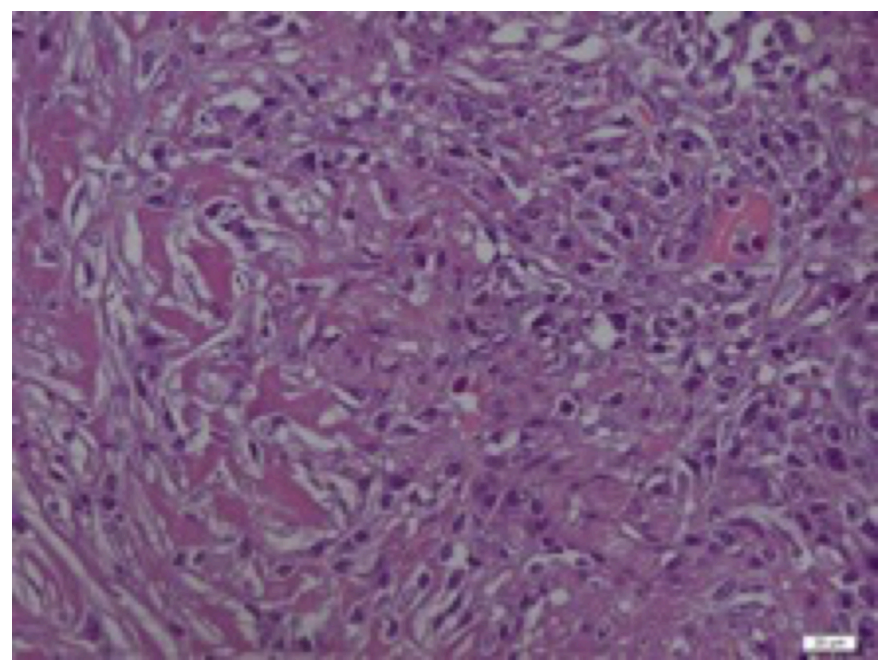

Figure 2: Light microscopy showing dense lymphoplasma cell infiltration andfibrosis. (H\&E, $\times 200)$.

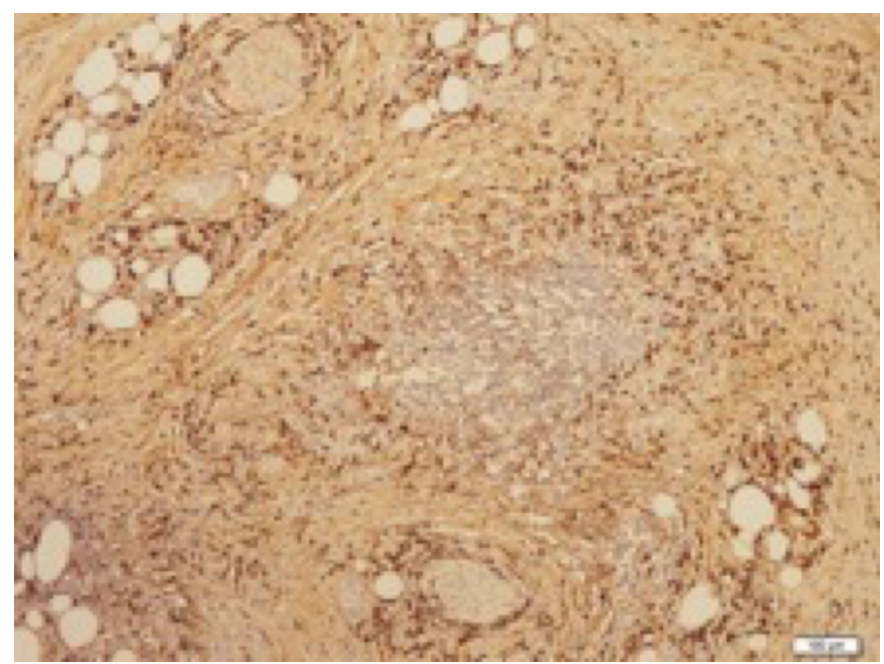

Figure 3: Immunostaining shows that many plasma cells are positive for IgG4. The ratio of the average number of IgG4-positive/IgG-positive cells after counting at 10 different high-power fields was $64 \%$. (IgG4 immunostaining, $\times 200$ ).

Pathological diagnosis was IgG4-related sclerosing mesenteritis (Figures 2 and 3). It was found that plasma IgG4 levels were increased to $457 \mathrm{mg} / \mathrm{dL}$ and $\mathrm{lgE}$, and eosinophil levels were normal. Postoperatively, obtained PET-CT was normal. The patient was started on $0.6 \mathrm{mg} / \mathrm{kg}$ methylprednisolone, which was gradually decreased and stopped on an average of three months. Control CTs of the patient revealed no disease recurrence. IgG4 level six months after the treatment was 190 $\mathrm{mg} / \mathrm{dL}$, and the patient remained well on follow-up for twoyears.

\section{DISCUSSION}

IgG4-RD is a chronic and autoimmune disease associated with increased IgG4 levels in multiple organs and/or tissues. It can affect many organs. Rate of mesenteritis in IgG4-RD varies between $1.6-7 \%$ in the literature. ${ }^{1,2}$ The disease may occur incidentally with radiological and pathological findings without clinical symptoms. In a large series of 235 cases, $71(30 \%)$ patients were asymptomatic and 96 (41\%) patients were detected with symptoms related to the affected organ. ${ }^{3}$ In the presentcase, it was diagnosed with a presentation with findings related to ureter pressure. The average age of onset of the disease is in the sixth or seventh decade, and it is more common in men. In some studies, it was seen in all age groups, including children. ${ }^{4}$

Serum IgG4 level, the most sensitive and specific test for lgG4-RD, is increased in most cases. Serum lgG4 levels $>135 \mathrm{mg} / \mathrm{dl}$ is important in diagnosis. However, in $30 \%$ of patients, serum IgG4 level may be normal. ${ }^{1,2}$ In this patient, serum IgG4 level was high and returned to normal after treatment. Allergic symptoms, and increased serum $\operatorname{lgE}$ levels in the blood and peripheral eosinophilia may also be seen in IgG4-RD. Eosinophil and IgE levels were normal in this patient.

Glucocorticoids are the primary treatment option and the response is generally good. The recommended treatment includes prednisolone at a dose of $0.6 \mathrm{mg} / \mathrm{kg} /$ day for two to four weeks and a dose reduction afterwards to $2.5-5 \mathrm{mg} /$ day formaintenance. It is recommended that maintenance therapy should be continued for at least three months to prevent relapse. ${ }^{2,6}$ In the present case, steroid treatment was continued for three months after surgery. No recurrence was detected at two years follow-up after treatment discontinuation. Immunosuppressive therapy, such as azathioprine or mycophenolate mofetil, may be considered in steroid-resistant cases. In patients with recurrent or refractory disease, rituximab or bortezomib treatments are also recommended. Anti-TNF $\alpha$ treatments have been tried in refractory cases. ${ }^{2,7}$ This case is of great interest in terms of progressive presentation under treatment with etanercept, an anti-TNF $\alpha$ agent, which is also used in the treatment.

The association of IgG4-RD with other rheumatological diseases is reported. In the cohort of Fernández-Codina et al. including 54 patients, four had RA, one had antineutrophil cytoplasmic antibodies (ANCA)-negative systemic vasculitis, and one had $\mathrm{AS}^{8}{ }^{8}$ In the French cohort, 25 patients had RA and one patient had $A S .{ }^{9}$ In the cohort of 235 cases, RA, antiphospholipid antibody syndrome, and ANCA-related vasculitis were detected in one case each, respectively. ${ }^{3}$ This study supports that association with AS is rare. In the literature, no case was observed to progress under antiTNF $\alpha$ treatment.

The association of IgG4-RD with AS is exceedingly rare in the litera- 
ture. IgG4-RD has not been observed in any AS patients under biological treatment. The authors presented thiscase because it is of interest that the patient presented with progressive disease under anti-TNF $\alpha$ treatment, which is also used in the treatment of the disease.

\section{ETHICALAPPROVAL:}

The study was approved by the Ethics Committee of University of Health Sciences, Umraniye Training and Research Hospital, Istanbul, Turkey.

\section{PATIENT'S CONSENT:}

Written informed consent form was obtained from the patient for the publication of the case details.

\section{CONFLICT OF INTEREST:}

The authors declared no conflict of interest.

\section{AUTHORS' CONTRIBUTION:}

OP, SOS, EEY: Conception or the design of the manuscript, acquisition, analysis and interpretation of the data.

All authors have participated in drafting the manuscript; whereas, Ozlem Pehlivan and Sibel Ocak Serin revised it critically.

\section{REFERENCES}

1. Zihni FY, Keser G. Immunoglobulin G4-related disease. J Turk Soc Rheumatol 2014; 6:53-61. doi:10.2399/raed. 14.10820.

2. Kamisawa T, Zen Y, Pillai S, Stone JH. IgG4-related disease. Lancet 2015; 385:1460-71. doi:10.1016/S0140-
6736(14)60720-0.

3. Inoue D, Yoshida K, Yoneda N, Ozaki K, Matsubara T, Nagai $\mathrm{K}$, et al. IgG4-related disease: Dataset of 235 consecutive patients. Medicine 2015; 94:e680. doi:10.1097/MD.00000 00000000680.

4. Chen LYC, Mattman A, Seidman MA, Carruthers MN. IgG4related disease: What a hematologist needs to know. Haematologica 2019; 104:444-55. doi:10.3324 /haematol.2018.205526.

5. Deshpande V, Zen Y, Chan JK, Yi EE, Sato Y, Yoshino T, et al. Consensus statement on the pathology of IgG4-related disease. Mod Pathol 2012; 25:1181-92. doi: 10.1038/ modpathol.2012.72.

6. Lanzillotta M, Mancuso G, Della-Torre E. Advances in the diagnosis and management of IgG4 related disease. BMJ 2020; 369:m1067. doi: 10.1136/bmj.m1067.

7. Wallace ZS, Deshpande V, Mattoo H, Mahajan VS, Kulikova M, Pillai S, et al. IgG4-Related disease: Clinical and laboratory features in one hundred twenty-five patients. Arthritis Rheumatol 2015; 67:2466-2475. doi:10.1002/art.39205.

8. Fernández-Codina A, Martínez-Valle F, Pinilla B, López C, DeTorres I, Solans-Laqué R, et al. IgG4-Related disease: Results from a multicenter Spanish registry. Medicine 2015; 94: e1275. doi:10.1097/MD.0000000000001275.

9. Ebbo $M$, Daniel L, Pavic M, Sève $P$, Hamidou $M$, Andres $E$, et al. IgG4-related systemic disease: Features and treatment response in a French cohort: Results of a multicenter registry. Medicine 2012; 91: 49-56. doi:10.1097/MD. ob013e3182433d77. 